\title{
Carolyne LARRINGTON, Winter is coming : les racines médiévales de Game of Thrones
}

\section{Tasnime Ayed}

\section{(2) OpenEdition}

\section{Journals}

Édition électronique

URL : https://journals.openedition.org/ccm/7604

DOI : $10.4000 / \mathrm{ccm} .7604$

ISSN : 2119-1026

\section{Éditeur}

Centre d'études supérieures de civilisation médiévale/Université de Poitiers

\section{Édition imprimée}

Date de publication : 1 juin 2021

Pagination : 168-170

ISBN : 978-2-490783-09-0

ISSN : 0007-9731

\section{Référence électronique}

Tasnime Ayed, "Carolyne LARRINGton, Winter is coming : les racines médiévales de Game of Thrones », Cahiers de civilisation médiévale [En ligne], 254 | 2021, mis en ligne le 01 juin 2021, consulté le 22 novembre 2022. URL : http://journals.openedition.org/ccm/7604 ; DOI : https://doi.org/10.4000/ccm. 7604

\section{(c)}

Creative Commons - Attribution - Pas d'Utilisation Commerciale - Pas de Modification 4.0 International - CC BY-NC-ND 4.0

https://creativecommons.org/licenses/by-nc-nd/4.0/ 
Carolyne LARRINGTON, Winter is coming : les racines médiévales de Game of Thrones, Antoine Bourguilleau (trad.), Paris, Passés Composés/Humensis, 2019.

Rédigé en 2015 par Carolyne Larrington, professeure universitaire de littérature européenne médiévale au St John's College à Oxford et spécialiste de légendes arthuriennes et nordiques, Winter is coming : les racines médiévales de Game of Thrones paraît en 2019 aux éditions Passés Composés en France. La traduction française d'Antoine Bourguilleau introduit les lecteurs francophones à l'univers médiévalisant du Trône de Fer, cycle romanesque et série télévisée. L'a. établit, en effet, des parallèles entre le Moyen Âge et les différentes configurations historiques, géographiques, sociales et politiques de l'univers fantasy crée par George R. R. Martin, et tente « d'expliquer le Monde connu, ses coutumes, ses habitants, ses luttes de pouvoir, ses religions et ses cultures, par le biais de la lentille médiévale » (p. 29). Le corpus étudié couvre les cinq romans et les cinq premières saisons de leur adaptation télévisée, l'a. considérant l'ensemble comme une saga homogène, tout en signalant ponctuellement certains écarts.

Winter is coming s'ouvre avec une «Introduction » où $\mathrm{C}$. Larrington expose le cadre général dans lequel l'univers du Trône de Fer se déploie. Plusieurs éléments font « écho à des organisations sociales et culturelles que l'on retrouve en Europe et en Asie centrale au Moyen Âge » (p. 20). Mais, « soumis au filtre de l'imagination de Martin [...], les faits historiques sont transformés en quelque chose de plus riche, de plus étrange, de plus archétypal » (p. 20-21). Les cinq chapitres suivants s'organisent selon l'agencement géographique du Monde connu, proposant au lecteur un voyage où l'auteure-guide dévoile comment les composantes médiévales sont ciselées.

«Le Centre», premier chapitre, explicite l'articulation des concepts généraux et fondamentaux de la saga. L'a. s'intéresse « aux vieux principes bien établis qui affectent particulièrement les individus : généalogie, maisons, honneur, réputation, tradition orale et discours, visions très patriarcales de l'amour, du genre et du sexe » (p. 31-32) avant de se pencher sur « les structures sociales élargies : hospitalité, justice et vengeance, guerre et armement » (p. 32). En Westeros comme en Europe médiévale occidentale, l'ancienneté de la lignée noble confère à la descendance légitime dignité et autorité. Les récits médiévaux, tels Beowulf ou Le Morte d'Arthur, sont également imprégnés de ces valeurs. Le Conte de Gamelyn relate le conflit violent de deux frères s'affrontant pour les droits de succession, à l'image de Stannis et Renly Baratheon. La primogéniture en vigueur en Westeros " prend forme au $\mathrm{Xl}^{\mathrm{e}}$ siècle en Europe » (p. 46) afin de lutter contre l'effritement des territoires et du pouvoir, mais génère des guerres internes entre fratries. À ce titre, la région de Dorne est exceptionnelle. Elle assure aux femmes liberté et égalité alors que le reste des « femmes de Westeros et d'Essos sont, comme leurs sœurs du Moyen Âge, assujetties aux règles du patriarcat » (p. 48). La fonction des dames aristocrates dans l'histoire médiévale et dans le Monde connu est d'unifier et de renforcer leurs clans à travers le mariage. Mais « à partir du moment où l'Église commence à insister sur l'importance du consentement de la femme lorsqu'une alliance est contractée, l'idée que le respect mutuel et l'affection font des mariages plus solides et plus heureux pour les deux époux gagne du terrain. Les maris qui partent en croisade, parfois pendant une décennie, doivent être certains que leurs épouses sauront gérer leur domaine en leur absence; et l'influence dissimulée des épouses et des mères peut parfois changer le destin des nations » (p. 50). Telles Cersei Lannister et Catelyn Stark, impliquées dans la guerre des Cinq Rois, Aliénor d'Aquitaine lance la première étincelle de la guerre de Cent Ans et Marguerite d'Anjou joue un rôle capital durant la guerre des Deux-Roses. L'a. explique ensuite la notion d'hospitalité, code moral protégeant à la fois l'hôte et l'invité. « Mais [ce] n'est pas toujours un concept aussi simple dans le Monde connu, comme dans les récits médiévaux » (p. 56), où la trahison est suivie de conséquences graves. Les noces pourpres font ainsi écho au Conte de l'homme de loi de Chaucer et à la légende historique du Black dinner. Le récit de vengeance est un trope médiéval, prenant parfois place dans le cadre d'un procès. En matière de justice, l'univers du Trône de Fer suit l'exemple de la plupart des pays de l'Europe médiévale, « la loi c'est la loi du roi, et ses représentants la font appliquer sur-lechamp » (p. 61). Mais, comme Tyrion Lannister face à l'accusation de régicide, les inculpés peuvent faire appel au duel judiciaire, coutume médiévale issue des régions germaniques et dont les récits médiévaux, 
de Chrétien de Troyes par ex., regorgent. Par ailleurs, dans l'univers imaginaire de G. R. R. Martin, les dragons représentent l'élément magique central. Créatures imbattables, arme redoutable aux mains de Daenerys Targaryen, ils perturbent l'équilibre politique de Westeros et peuplent les fictions et légendes médiévales des traditions germaniques, du folklore britannique et des sagas nordiques.

Le deuxième chapitre se focalise sur « le Nord», une région qui « tente sans cesse de maintenir un fragile équilibre entre un Sud plus politique et les créatures étranges que l'on trouve après le Mur » (p. 76). Winterfell est le foyer du pouvoir de la maison Stark gouvernant le Nord. Son architecture « rappelle celle des châteaux concentriques normands bâtis par Édouard I ${ }^{\mathrm{er}}$, particulièrement au pays de Galles » (p. 78). Lord Eddard Stark exerce un pouvoir seigneurial suivant le modèle anglo-saxon, référant principal de l'imaginaire nordien. Les loups-géants sont, ainsi, inspirés d'une race préhistorique. « Symbole ancien de terreur dans l'imaginaire occidental » (p. 81), l'animal est lié à la guerre, à l'exil et au concept de métamorphose dans la pensée nordique et la tradition anglo-saxonne, également habitée de géants sauvages, de corbeaux et de corneilles à la sagesse supérieure. Une corneille à trois yeux guide Bran Stark dans la découverte de ses pouvoirs liés aux anciens dieux. C. Larrington précise que « la religion des anciens dieux, celle des Premiers Hommes et de leurs descendants du Nord, est une vénération animiste des pouvoirs de la nature. À cet égard, elle n'est pas sans rappeler la religion pratiquée en Grande-Bretagne avant l'arrivée du christianisme, par les Bretons, telle que décrite par les historiens romains, et par les Anglo-Saxons » (p. 121). Au sud de Winterfell, alors que la lutte les Paludiers de la région du Neck rappelle « la résistance des habitants des Fens marécageux d'Est-Anglie, au nord-est de Londres, contre les envahisseurs normands » (p. 91), les Fer-nés des îles de Fer, basant leur puissance sur leurs navires de guerre et vivant principalement de pillage " ont beaucoup à voir avec les Vikings du $\mathrm{VIII}^{\mathrm{e}} \mathrm{au} \mathrm{XII}^{\mathrm{e}} \mathrm{s}$. [...] qui sèment la terreur en Europe » (p. 93). Au nord de Winterfell, se dresse le Mur, inspiré, comme l'affirme G. R. R. Martin, du mur d'Hadrien « construit pour protéger la partie la plus septentrionale de l'Empire romain des invasions des terribles Pictes et Scots » (p. 100). Châteaunoir est une des bases les plus importantes de la Garde de
Nuit, ordre militaire dédié à protéger le royaume contre les menaces d'au-delà du Mur. La fraternité s'apparente aux ordres religieux chrétiens. L'a. note que « le christianisme médiéval avait bien saisi l'avantage d'associer la puissance des armes à la poursuite d'objectifs particuliers en créant les ordres militaires » (p. 103), comme l'ordre des Templiers et les chevaliers Teutoniques avec lesquels la Garde de Nuit partage certaines valeurs. La région d'au-delà du Mur représente « le royaume de l'étrange, la terre de glace, un lieu où les anciennes traditions prévalent, où les contes $[\ldots]$ prennent réellement corps, où les coutumes et croyances du reste de Westeros ne semblent pas avoir cours » (p. 108). Nous y rencontrons les sauvageons, dont l'armement rudimentaire renvoie aux petits couteaux, haches et pierres utilisées durant la guerre civile en Islande au XIII ${ }^{e}$ s., mais également les Marcheurs blancs, spectres revenants et créatures de glace. «Avec leur malveillance, et leur invincibilité surnaturelles, les spectres rappellent les morts-vivants de la tradition nordique, les draugar » (p. 112) tandis que les Autres, capables de réanimer les morts font écho aux pouvoirs de la princesse Hild de la mythologie nordique et au chaudron magique de roi de Galle du Nord. Dans les légendes médiévales, « le pouvoir mythique de ressusciter ceux qui sont morts au combat est synonyme de terreur $»($ p. 111). Dans le Trône de Fer, les revenants renvoient à la mort, puissance implacable et incontrôlable.

L'a. poursuit son analyse à l' " Ouest» de Westeros où «se déroulent des intrigues politiques complexes typiques de la fin du Moyen Âge et où la cour, le Conseil restreint et la chevalerie jouent un rôle central. La religion des Sept y prospère, une religion qui n'est pas sans rappeler le catholicisme médiéval » (p. 127). Laid et inconfortable, le Trône de Fer illustre les difficultés de gouverner et incarne « la violence qui accompagne les transitions du pouvoir et la force qu'il faut employer pour le conserver » (p. 129). La cour regorge de personnages à résonance historique et légendaire, complexes, arrivistes et craignant de perdre leur autorité. L'organisation administrative des Sept Couronnes, gouvernées depuis la capitale Port-Réal, par un roi, une Main, et un Conseil restreint, est proche de « l'Angleterre de la fin du Moyen Âge, dirigée depuis Westminster » (p. 130). Les outils et les stratégies de guerre sont également similaires aux techniques utilisées par les armées européennes médiévales. Loin d'une substance 
surnaturelle, le feu grégeois utilisé durant la bataille de la Néra est une arme « développée vers 672 par les chimistes de Byzance, équivalents médiévaux de la Guilde, et utilisée avec des effets remarquables contre les flottes musulmanes » (p. 148). L'a. dresse, par la suite, les parallèles entre le Monde connu et l'Europe médiévale en matière de valeurs chevaleresques, notamment en lien avec les conditions d'emprisonnement, et de principes économiques. En effet, « les finances de la dynastie Baratheon ne sont pas sans rappeler celles de l'Angleterre et de la France médiévale. La Couronne était constamment en quête de moyens d'augmenter ses revenus, en levant de nouveaux impôts de différentes natures, afin de financer la guerre au cours des XIV et $\mathrm{XV}^{\mathrm{e}} \mathrm{s}$. » (p. 162-163). C. Larrington parcourt ensuite le Bief où les mestres de Villevieille, ressemblant à « l'Oxford du Moyen Âge » (p. 171), rappellent les clercs, ainsi que Peyredragon où Shôren Baratheon a survécu à la léprose, équivalent fictif de la lèpre ayant ravagé le nord de l'Europe médiévale.

Les quatrième et cinquième chapitres se situent dans un autre continent du Monde connu, «Au-delà du Détroit » et à l'« Est ». " Pour les habitants de Westeros, les villes et les peuples d'Essos sont étranges, exotiques et même barbares; leurs langages sont obscurs, leurs coutumes incompréhensibles » (p. 76). La ville de Braavos, d'inspiration méditerranéenne, fonde sa puissance sur les échanges commerciaux et monétaires. Sa Banque de Fer s'apparente à la banque de Venise créée afin de remédier au besoin financier « engendré par le financement des croisades successives et la nécessité d'emprunter de l'argent pour permettre l'expansion de la république de Venise en Méditerranée orientale » (p. 185). Braavos est le siège de la guilde d'assassins des Sans-Visages, référant aux « Nizârites ismaïliens, une secte musulmane créée à la fin du $\mathrm{XI}^{\mathrm{e}}$ siècle en Perse et en Syrie » (p. 191), connus en Europe comme les Haschischins. Les compagnies mercenaires d'Essos renvoient également à la Méditerranée et la Compagnie blanche basée en Italie, dirigée par l'anglais John Hawkwood et redoutée dans toute l'Europe médiévale. C. Larrington souligne, par la suite, que les autres cités libres et la ville de Volantis reflètent un mélange de légendes à résonances médiévale et antique, et rapproche le culte de R'hllor du zoroastrisme, du catharisme et des autres sectes fondées sur des principes dualistes et considérées comme hérétiques au Moyen Âge. À l'est d'Essos, les parallèles se font principalement avec l'histoire médiévale asiatique. Les Dothrakis sont ainsi rapprochés des Mongols avec qui ils partagent brutalité et coutumes. Les cités de la baie des Serfs permettent à l'a. d'aborder les limites de la politique médiévale et de la conquête des terres étrangères. Daenerys Targaryen ne peut y asseoir son pouvoir à distance. « Les préoccupations de ces villes rappellent des problèmes bien médiévaux : d'abord les difficultés auxquelles est confrontée la dynastie angevine des Plantagenêt en Angleterre et en France au cours des siècles qui suivent la conquête normande; ensuite les difficultés des brefs États latins d'Orient, établis en Terre sainte auX XI et XII $\mathrm{e}$. » (p. 237). Mais alors que l'empire déchu de Valyria réfère aux cités antiques mythiques comme l'Atlantide ou historiques comme Pompéi, et aux cités bibliques maudites de Sodome et Gomorrhe, Qarth et l'Orient lointain, font écho aux stéréotypes de l'« orientalisme» (p. 260). Leurs vêtements colorés, épices précieuses, codes moraux flexibles, traditions barbares et pratiques magiques caractéristiques renvoient à une définition de « la culture orientale selon des normes occidentales, un occident capable de s'émerveiller et de mépriser l'Orient tout en même temps » (p. 260), à l'image des personnages westérossiens du Trône de Fer.

L'ouvrage se clôt sur un « Épilogue » où l'a. abandonne le Monde connu pour essayer d'esquisser une fin potentielle à la saga. Elle se base sur le récit de la légende d'Havelok le Danois, « le triomphe du roi légitime sur l'usurpateur [étant] un thème populaire dans l'Europe médiévale » (p. 264). Nous saisissons, ainsi, au bout du voyage, comment le monde imaginé par G. R. R. Martin donne vie au médiéval et le réactualise, son œuvre reflétant les cultures, croyances et pratiques de l'époque. Loin d'un exposé des sources d'inspirations directes de G. R. R. Martin, l'étude de C. Larrington souligne les similitudes entre l'imaginaire médiéval ou médiévalisant et l'Histoire. L'a. explicite à la fois la richesse thématique du Trône de Fer et les spécificités des légendes médiévales, leur construction, leur variabilité et leur résonance. Se prêtant aussi bien à la lecture curieuse d'un passionné de la série télévisée qu'à celle plus savante d'un public érudit, Winter is coming, est une véritable introduction à la recherche sur le médiévalisme dans l'œuvre de G. R. R. Martin et une référence académique incontournable pour en approfondir l'étude.

Tasnime AYED

Université de La Manouba, Tunisie LR18ES12 - ATTC / EA 4028 -

Textes et Cultures 